\title{
A complementary transposon tool kit for Drosophila melanogaster using $P$ and piggyBac
}

\author{
Stephen T Thibault ${ }^{1}$, Matthew A Singer ${ }^{1,2}$, Wesley Y Miyazaki ${ }^{1}$, Brett Milash ${ }^{1,2}$, Nicholas A Dompe ${ }^{1}$, \\ Carol M Singh ${ }^{1,2}$, Ross Buchholz ${ }^{1}$, Madelyn Demsky ${ }^{1}$, Robert Fawcett ${ }^{1}$, Helen L Francis-Lang ${ }^{1}$, Lisa Ryner ${ }^{1}$, \\ Lai Man Cheung ${ }^{1}$, Angela Chong ${ }^{1}$, Cathy Erickson ${ }^{1}$, William W Fisher ${ }^{1}$, Kimberly Greer ${ }^{1}$, Stephanie R Hartouni ${ }^{1}$, \\ Elizabeth Howie $^{1}$, Lakshmi Jakkula ${ }^{1}$, Daniel Joo ${ }^{1,2}$, Keith Killpack ${ }^{1}$, Alex Laufer ${ }^{1,2}$, Julie Mazzotta ${ }^{1}$, \\ Ronald D Smith ${ }^{1}$, Lynn M Stevens ${ }^{1}$, Christiana Stuber ${ }^{1}$, Lory R Tan ${ }^{1}$, Richard Ventura ${ }^{1}$, Alesa Woo ${ }^{1}$, \\ Irena Zakrajsek ${ }^{1,2}$, Lora Zhao ${ }^{1}$, Feng Chen ${ }^{1}$, Candace Swimmer ${ }^{1}$, Casey Kopczynski ${ }^{1,2}$, Geoffrey Duyk ${ }^{1}$, \\ Margaret L Winberg ${ }^{1} \&$ Jonathan Margolis ${ }^{1}$
}

With the availability of complete genome sequence for Drosophila melanogaster, one of the next strategic goals for fly researchers is a complete gene knockout collection. The $\boldsymbol{P}$ element transposon ${ }^{1}$, the workhorse of $D$. melanogaster molecular genetics, has a pronounced nonrandom insertion spectrum $^{2}$. It has been estimated that $87 \%$ saturation of the $\sim 13,500$-gene complement of $D$. melanogaster ${ }^{3}$ might require generating and analyzing up to 150,000 insertions $^{2}$. We describe specific improvements to the lepidopteran transposon piggy Bac $^{4}$ and the $\boldsymbol{P}$ element that enabled us to tag and disrupt genes in $D$. melanogaster more efficiently. We generated over $\mathbf{2 9 , 0 0 0}$ inserts resulting in $\mathbf{5 3} \%$ gene saturation and a more diverse collection of phenotypically stronger insertional alleles. We found that piggyBac has distinct global and local genetagging behavior from that of $\boldsymbol{P}$ elements. Notably, piggyBac excisions from the germ line are nearly always precise, piggyBac does not share chromosomal hotspots associated with $P$ and piggy $B$ ac is more effective at gene disruption because it lacks the $\boldsymbol{P}$ bias for insertion in $5^{\prime}$ regulatory sequences.
We incorporated three technological improvements to increase the efficiency of transposon mutagenesis in D. melanogaster, with an emphasis on generating stronger loss-of-function alleles. First, we used an additional mobile genetic element. Alternative transposons have been used in D. melanogaster ${ }^{5}$, but for various technical reasons, none had been applied for large-scale gene knockouts when we began our studies. We selected piggyBac as the most promising transposon for this application. Owing to the low remobilization rates observed with a heat shock-inducible transposase (data not shown), we reengineered the transposase with a constitutive promoter and germline-stabilizing $3^{\prime}$ untranslated region. With this construct, we and others ${ }^{6,7}$ observed remobilization frequencies of $60-80 \%$ (data not shown). Second, we constructed a series of different $P(\mathrm{XP})$ and piggyBac $(\mathrm{PB}, \mathrm{RB}, \mathrm{WH})$ vectors carrying 'splice-trap' and transcriptional silencing elements (Supplementary Fig. 1 online) for more effective gene disruption. Third, although previous screens typically mobilized transposons in the male germ line, differences in transposon activity between male and female germ lines ${ }^{8}$ led us to use the female germ line, with the hope of altering the spectrum of genes accessible to tagging. An ongoing challenge for

Table 1 Distribution of $P$ and piggyBac transposons across the genome

\begin{tabular}{|c|c|c|c|c|c|c|c|c|}
\hline \multirow[b]{2}{*}{ Transposon } & \multicolumn{8}{|c|}{ Chromosome arma } \\
\hline & $x$ & $2 \mathrm{~L}$ & $2 \mathrm{R}$ & $3 \mathrm{~L}$ & $3 R$ & 4 & $U^{b}$ & Total \\
\hline$P$ element XP & 1,803 & 1,280 & 1,528 & 1,800 & 1,848 & 22 & 34 & 8,315 \\
\hline piggyBac PB & 658 & 1,018 & 1,130 & 1,582 & 1,931 & 45 & 434 & 6,798 \\
\hline piggyBac RB & 814 & 769 & 820 & 892 & 965 & 53 & 35 & 4,348 \\
\hline piggyBac WH & 1,470 & 1,264 & 1,172 & 1,407 & 1,604 & 77 & 85 & 7,079 \\
\hline
\end{tabular}

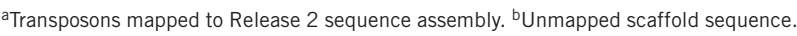

${ }^{1}$ Exelixis, 170 Harbor Way, South San Francisco, California 94083-0511, USA. 2Present addresses: Chemicon International, 28820 Single Oak Drive, Temecula, California 92590, USA (M.A.S.); Huntsman Cancer Institute, 2000 Circle of Hope, University of Utah, Salt Lake City, Utah 84132, USA (B.M.); EnVivo Pharmaceuticals, 480 Arsenal Street, Building 1, Watertown, Massachusetts 02472, USA (C.M.S.); Metabolex, 3876 Bay Center Place, Hayward, California 94545, USA (D.J.); Renovis, Two Corporate Dr., South San Francisco, California 94080, USA (A.L.); Genentech, 1 DNA Way, South San Francisco, California 94080, USA (I.Z.); Ercole Biotech, 7030 Kit Creek Road, P.O. Box 12295, Research Triangle Park, North Carolina 27709, USA (C.K.). Correspondence should be addressed to S.T.T. (thibault@exelixis.com). 
Table 2 Summary of deficiency complementation and excision of lethal piggyBac insertions

\begin{tabular}{|c|c|c|c|c|c|c|}
\hline $\begin{array}{c}\text { Lethal } \\
\text { piggyBac }{ }^{\mathrm{a}}\end{array}$ & Chromosome & $\begin{array}{c}\text { Noncomplementing } \\
\text { deficiency }^{\mathrm{b}}\end{array}$ & $\begin{array}{l}\text { Independent } \\
\text { excisions }\end{array}$ & $\begin{array}{l}\text { Viable } \\
\text { excisions }\end{array}$ & $\begin{array}{c}\text { Excisions complementing } \\
\text { deficiency }\end{array}$ & $\begin{array}{l}\text { Precise molecular } \\
\text { excisions }\end{array}$ \\
\hline c00080 & 2 & B-3084, Df(2L)ast2 & 10 & $10 / 10$ & $10 / 10$ & $10 / 10$ \\
\hline c00093 & 2 & B-442, $D f(2 R) C X 1$ & 10 & $10 / 10$ & $10 / 10$ & $10 / 10$ \\
\hline c00146 & 2 & B-384, $D f(2 L) N 22-5$ & 10 & $10 / 10$ & $10 / 10$ & $10 / 10$ \\
\hline c00221 & 2 & B-1006, $D f(2 R)$ nap 1 & 10 & $10 / 10$ & $10 / 10$ & $10 / 10$ \\
\hline c00119 & 3 & B-1910, Df(3R)TI-P & 10 & $10 / 10$ & $10 / 10$ & $10 / 10$ \\
\hline c00083 & 3 & B-3011, Df(3R)Cha7 & 5 & $5 / 5$ & $5 / 5$ & $5 / 5$ \\
\hline c00136 & 2 & B-3138, Df(2L)b87e25 & NA & & & \\
\hline c00014 & 3 & B-1011, Df(3R)faf-BP & NA & & & \\
\hline c00064 & 3 & B-3007, Df(3R)ry615 & NA & & & \\
\hline c00213 & 3 & B-3627, $D f(3 L) 31 A$ & NA & & & \\
\hline
\end{tabular}

aExelixis stock number. ${ }^{b}$ Bloomington Drosophila Stock Center number and deficiency name. NA, not applicable.

fruit fly researchers is that mutants and transgenic strains come from a variety of genetic backgrounds that may harbor pre-existing mutations ${ }^{9}$, thus precluding or complicating highly sensitized or behavioral screens. We generated all our transposon reagents and associated stocks in a freshly derived isogenic background; the insertion collection reported here is fully isogenic.

With these new tools and strategies we generated 29,682 piggyBac and XP insertion lines, of which $89 \%$ were molecularly placed on the genome (Table 1). We observed higher frequencies of recessive lethal mutations for both XP (17\%) and piggyBac (22\%) than the 10-15\% reported from previous $P$ screens ${ }^{10}$. Molecular and genetic analysis suggests that this was not simply due to multiple insertions. Eightynine percent of the lines yielded unique flanking sequence by inverse PCR. In addition, 97 of 100 piggyBac lines analyzed by genomic Southern blotting had single insertions (data not shown). The increased lethal frequency observed for piggyBac was not the result of elevated background mutagenic effects ('hit and runs') at sites independent of the final insertion. Because we started with a clean isogenic strain, we were able to assess the background mutagenic rate with high accuracy using the following tests. We selected ten lethal piggy Bac lines and confirmed that each did not complement a corresponding deficiency (Table 2), indicating these mutations are at least closely linked to the insert. We then used six of these lines to generate 55 excision alleles; each was molecularly confirmed as a precise event that also reverted the chromosome to a viable phenotype (Table 2). Previous studies also found a majority of molecularly precise excision events,

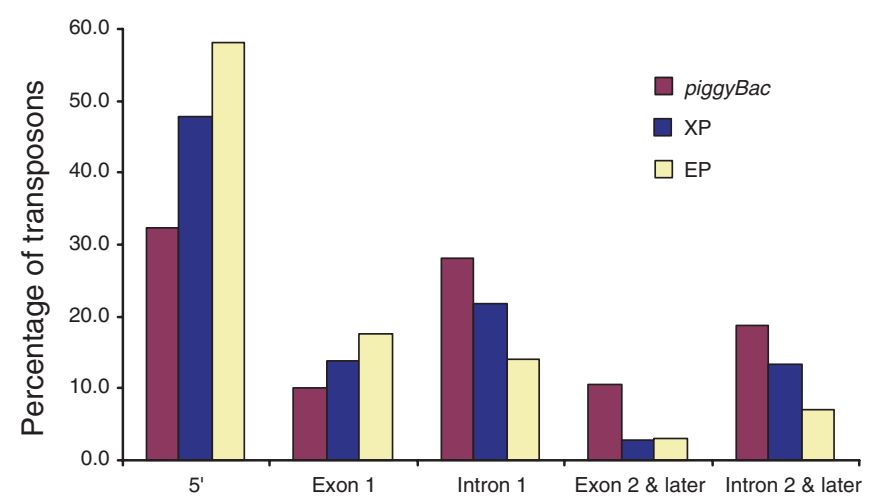

Figure 1 Distribution within genes of 6,196 piggyBac elements, 2,462 XP elements and 1,109 EP ${ }^{11}$ transposons tagging DGC ( $\left.r 1.0\right)$ genes. 5' of start $=1,000-\mathrm{bp}$ window upstream of the transcriptional start. although lethal alleles (generated in a nonisogenic background) were not always reverted. Additionally, separate genetic screens using our collection found that only $0.3-0.5 \%$ of piggyBac stocks contained unlinked background mutations (H.L.F.-L., unpublished data); thus; hit and run events do not account for the high percentage of recessive lethal piggyBac insertions. Finally, secondary mutations may arise if transposase expression activates cryptic elements or induces genomic instability. The insertion and transposase strains have been stable for 4 years with no observed breakdown.

Taken together, these data support the conclusion that piggyBac acts as an effective mutagen and that the mutations observed are directly caused by the transposon insertion. The higher frequency of lethal mutations generated by piggyBac versus $P$ cannot be explained by secondary mutations but must result from some other property of the transposon, such as its insertion pattern or the ability of the transposon itself to locally perturb gene function. To investigate these possibilities, we compared the local and global patterns of piggyBac and $\mathrm{XP}$ insertions.

After associating insertions with a highly curated set of 5,849 genes (the Berkeley Drosophila Genome Project's Drosophila Gene Collection (DGC) r1.0), we determined their relative positions within the genes (Fig. 1). We also analyzed an unbiased public set of $P$ insertions (EP element ${ }^{11}$ ) for comparison. We found that a larger fraction of piggyBac elements inserted after the transcriptional start site $(67.5 \%$ versus $52.1 \%$ of XP and $41.9 \%$ of EP lines). Unlike $P$, which preferentially inserts at the $5^{\prime}$ end of genes ${ }^{12}$, only one-third of piggyBac inserts were found upstream of the transcriptional start site (Fig. 1). The XP collection was also less biased than EP by this metric, possibly because we used female dysgenesis in our screen. We also observed that piggyBac inserted into coding exons at a higher rate than either XP or EP. Excluding first exons, piggyBac tagged remaining exons more than three times more frequently. Because initial exons in D. melanogaster are often non-protein coding, insertions in later exons are more likely to fully disrupt gene function by interrupting the open reading frame. Intronic insertions may also be disruptive by interfering with regulatory sequences or proper gene splicing ${ }^{13,14}$. Thus, because piggyBac insertions occur more frequently between transcriptional start and stop, they should create null alleles more commonly.

Examining molecular placements identified hot-spot regions for both XP and piggyBac (Fig. 2). Defining a hot spot as a 50-kb interval containing 30 or more insertions, we found $23 \mathrm{XP}$ hot spots (Supplementary Table 1 online). In contrast, from more than twice as many piggyBac insertions, we found only 26 piggyBac hot spots (Supplementary Table 1 online), none of which overlapped XP hot 
Left Arm (2L)

Figure 2 Distribution of XP $(2,661)$ and piggyBac $(6,765)$ inserts across the left and right arms of $D$. melanogaster chromosome 2. Chromosome sequence was binned into 50-kb intervals and the number of transposons in each bin is plotted. Hot-spot and cold-spot regions are apparent for both transposons. All hot spots are nonoverlapping between $P$ and piggyBac. Results were similar across remaining chromosomes.

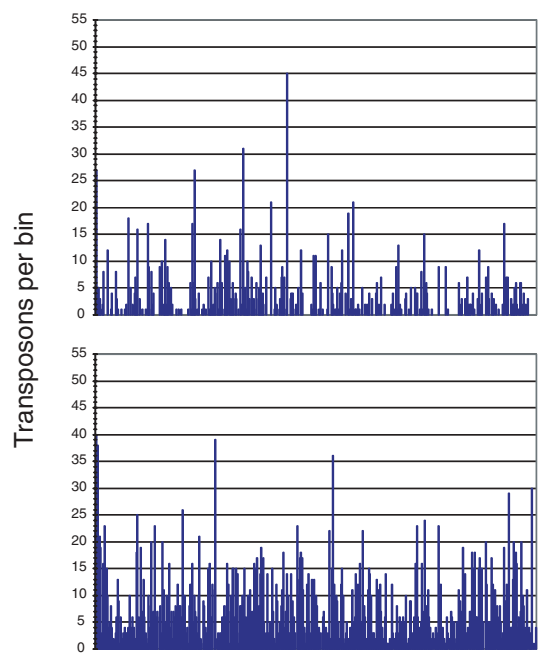

Right Arm (2R)

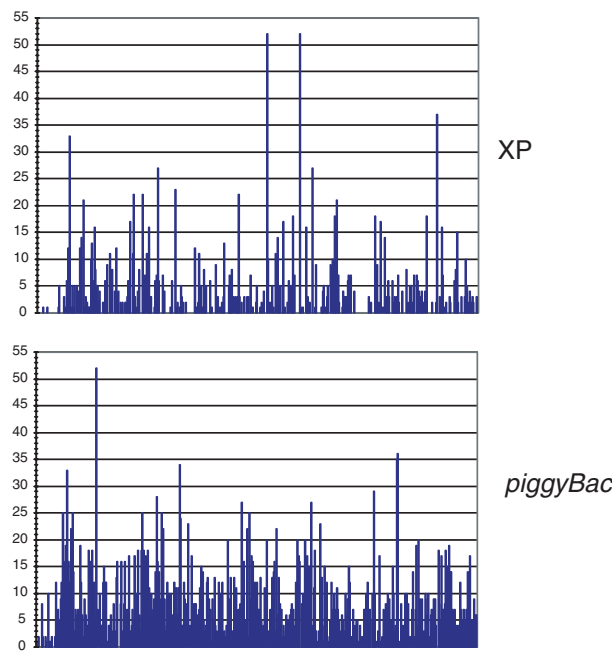

spots. The mean number of piggyBac insertions per hot-spot bin (38) was also less than that for XP (43). Neither transposon had a random (Poisson) distribution (Fig. 3), but the spectrum of piggyBac insertions was different from and broader than the spectrum of XP insertions.

To assess relative gene tagging frequencies for $P$ and piggyBac, we plotted the cumulative number of DGC genes tagged against recovery of new insertions (Fig. 4). piggyBac was much more efficient, yielding a high rate of new hits even after 17,000 insertions. Therefore, to achieve $87 \%$ gene saturation with piggyBac should require substantially fewer insertions than to do so using $P$ alone, perhaps 75,000-100,000. Our combined piggyBac and XP collection tagged $53 \%$ of the DGC genes and, by extension, all D. melanogaster genes.

After generating and characterizing thousands of piggyBac and $P$ element insertions, we find piggyBac to be an efficient and practical gene tagging system in $D$. melanogaster on par with the $P$ element.
These reagents and the associated transposon tool kit will be a useful complement to existing $D$. melanogaster gene knockout resources. In fact, this collection has already been extensively used by us and our collaborators for biological analysis of gene function in pharmaceutically relevant disease pathways ${ }^{15,16}$.

\section{METHODS}

D. melanogaster strains and plasmid vectors. We maintained flies on standard cornmeal-molasses-agar medium at $25{ }^{\circ} \mathrm{C}$ (ref. 9). We used three classes of piggyBac vectors and one $P$ element in the screen (Supplementary Fig. 1 online). The piggyBac vector $(\mathrm{PB})$ is the simplest, comprising a complete piggyBac transposon with the open reading frame interrupted by the D. melanogaster gene mini-white $\left(w^{+\mathrm{mC}}\right)^{17}$ flanked by short (48-bp) FRT recombination sites from the yeast $2 \mu$ plasmid $^{18}$. The piggyBac vector (RB) contains the mini-white marker gene and a single long (199-bp) FRT site. Because exons in $P$ elements can disrupt gene function by acting as ectopic splice acceptors ${ }^{13,14}$, we designed the RB vector with an exon from the D. melanogaster gene Rbpl (ref. 19) as a second splice trap a

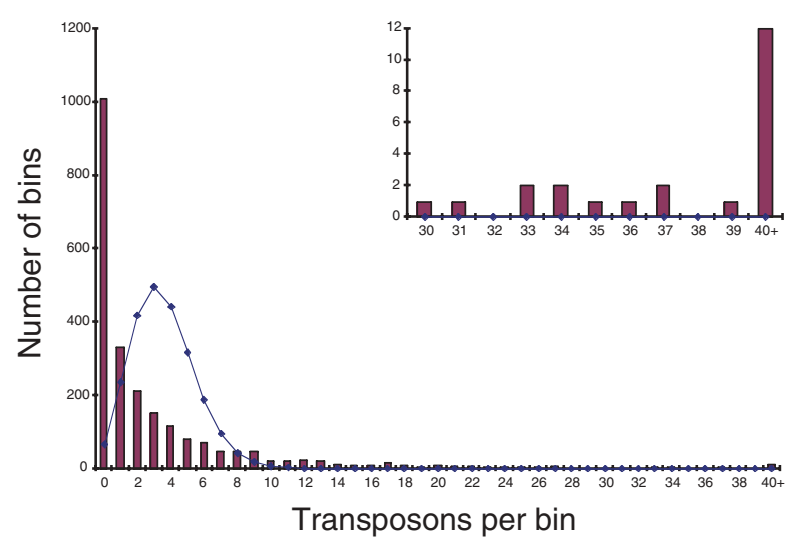

b

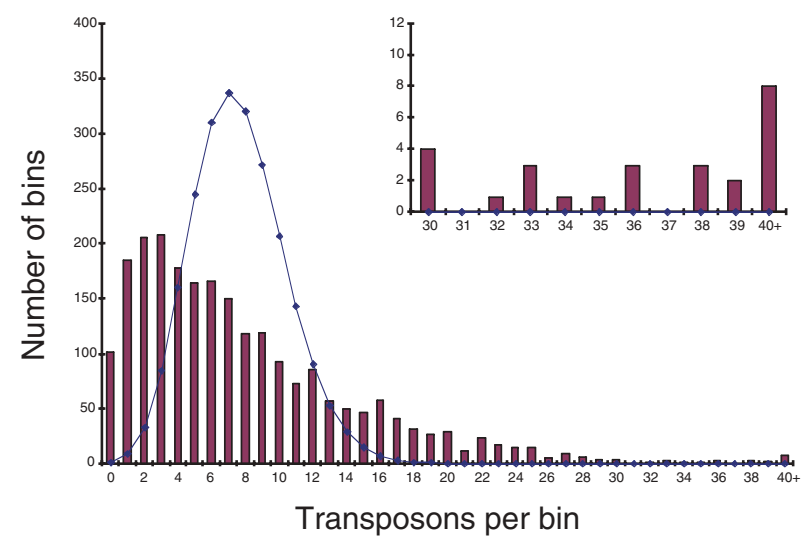

Figure 3 The assembled D. melanogaster genome sequence was divided into 50-kb bins and the number of transposons in each bin is represented as a histogram for XP (a) and all piggyBac insertions (b). Overlaid on each histogram is a line representing the predicted Poisson distribution for 8,281 XP and 17,671 piggyBac inserts (transposons placed on unassembled Chromosome $U$ are excluded from this analysis). Both XP and piggyBac distributions were significantly different from Poisson ( $\chi^{2}$ analysis, $P<0.001$ ). The number of bins containing 30 or more transposons is highlighted in the inset histograms. $\mathrm{XP}$ and piggyBac elements had comparable numbers of hot spots, although more than twice as many piggyBac inserts were generated. 


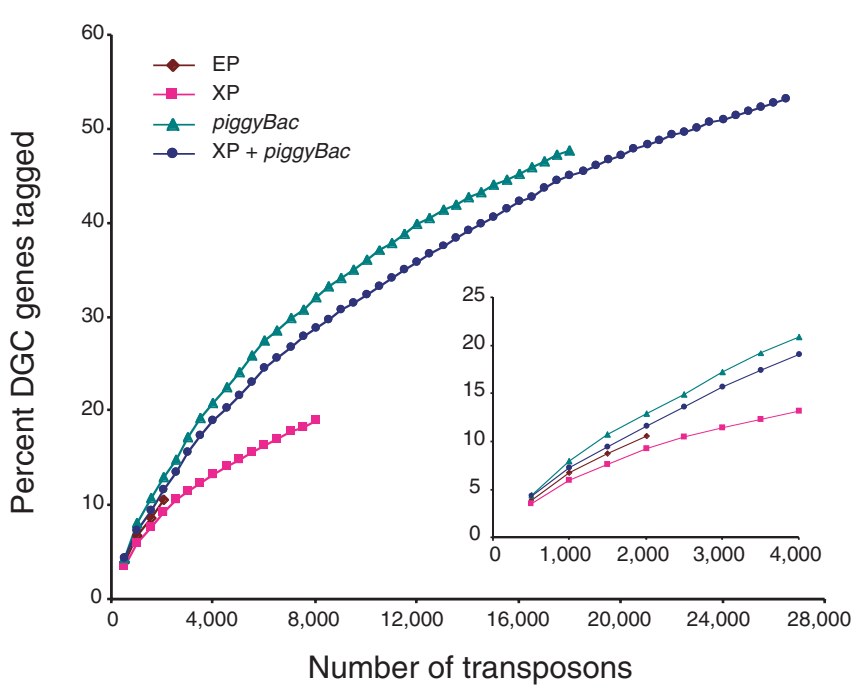

Figure 4 Gene-tagging frequency of $P$-element (XP) and piggyBac transposons. The EP ${ }^{11}$ set is included for comparison. Percentage of DGCtagged genes was plotted by random sampling of the set with increasing numbers of transposons. At an equal number of transposons $(8,000)$, piggyBac tagged $69 \%$ more genes than XP. The combined collection of $26,540 \mathrm{XP}$ and piggyBac molecularly localized insertions tagged $\sim 53 \%$ of DGC genes. The initial gene tagging rate is highlighted in the inset graph.

in the opposite orientation of mini-white. The piggyBac vector $(\mathrm{WH})$ contain the gene mini-white, a long FRT site, $\mathrm{Su}(\mathrm{Hw})$ insulator sequence ${ }^{20}$ and a terminal UAS site for Gal4-driven misexpression of adjacent genes ${ }^{11}$. The $P$ element (XP) used in our screen contains the gene mini-white, $\mathrm{Su}(\mathrm{Hw})$ insulator sequences and dual terminal UAS sites with one flanked by long FRT sites. In this issue, Parks et al. ${ }^{21}$ describe the use of FRT sites within XP, RB and WH for FLPmediated recombination ${ }^{22}$ between two insertions, enabling a rapid method for generating molecularly defined deficiencies.

We cloned piggyBac transposase sources into the improved $P$-element vector $\mathrm{pExPress}$ (derived from $\mathrm{pCasPeR}$ ) and introduced them into D. melanogaster by standard methods. We remobilized transposase sources onto the $\mathrm{CyO}$ balancer chromosome and characterized them for activity before selecting the isolates used in our screen. We constructed the inducible piggyBac transposase by placing the piggyBac coding sequence under control of the D. melanogaster Hsp70 promoter and regulatory sequences. We constructed the constitutive piggyBac transposase by cloning the piggyBac open reading frame under control of the $D$. melanogaster $\alpha$ Tub $84 B$ promoter ${ }^{23}$ into a variant of $\mathrm{pExPress}$ in which the gene mini-white is flanked by short FRT sequences. We further modified this piggyBac transposase source by adding the $f_{\mathcal{s}}(1) \mathrm{K}_{10} \mathrm{3}^{\prime}$ untranslated region to lend germline stability to the transcript ${ }^{24,25}$. The FRT-flanked gene mini-white allowed for FLP-mediated removal of the marker gene from transformed lines to generate a $w^{-}$piggyBac transposase strain.

Remobilization. We remobilized the PB element using Hsp70:piggyBac transposase from a single ammunition element on either the $\mathrm{X}$ or third chromosome. We induced transposase expression by immersing bottles in a circulating $37^{\circ} \mathrm{C}$ water bath for a daily (days 3-10 after egg-laying) 1-h heat shock. We outcrossed the resulting dysgenic males to an isogenic $w$ strain. New insertions were identified on the basis of a change in eye color (third chromosome ammunition) or the appearance of $w^{+}$male progeny (X chromosome ammunition). We remobilized both RB and WH elements using the constitutive $\alpha-1$ tubulin:piggyBac transposase source. We remobilized the RB element from a single $\mathrm{X}$ chromosome insertion in dysgenic males and the WH element from a single ammunition element on the Binsinscy balancer chromosome in dysgenic females. We outcrossed dysgenic males or virgin females in vials to the isogenic $w$ strain and selected new hops in the following generation. Only a single new insertion was retained per vial. For the $P$ element, XP, we selected an easily mobilized ammunition element among inserts hopped onto the Binsinscy balancer. New insertions were collected in vials from dysgenic females using the standard chromosomal source of transposase, $\Delta 2-3$ (ref. 26). All lines were mapped to a chromosome by standard genetic methods, examined for homozygous viability and used for recovery of flanking genomic sequence.

Localization by sequence. We obtained flanking genomic sequence from $5^{\prime}$ and 3' piggyBac ends by inverse PCR using a method adapted from J. Rehm (Berkeley Drosophila Genome Project). We purified DNA from five flies per line in 96-well format. DNA was digested separately with Sau3A1 (5') and Hin $\mathrm{P} 1\left(3^{\prime}\right)$ and then diluted and self-ligated. We obtained PCR products for piggyBac $5^{\prime}$ flanks by a nested reaction with primers $5 \mathrm{~F} 1$ and $5 \mathrm{R} 1$ followed by dilution (1:100) and a second PCR with primers 5F2 and 5R2. We obtained PCR products for $3^{\prime}$ flanks with nested primers $3 \mathrm{~F} 1$ and $3 \mathrm{R} 1$ for the first round and $3 \mathrm{~F} 2$ and $3 \mathrm{R} 2$ for the second round. Similarly, we recovered $5^{\prime}$ flanking sequence for XP with primers $51 \mathrm{~A}$ and $51 \mathrm{~B}$ for the first round and 52A and $52 \mathrm{~B}$ for the second round. For $3^{\prime} \mathrm{XP}$ inverse PCR, we used the primers $31 \mathrm{~A}$ and $31 \mathrm{~B}$ twice. We treated products with shrimp alkaline phosphatase and DNA exonuclease I. We determined sequences using fluorescent label dye-terminator chemistry (BigDye, ABI) with primers $\mathrm{pB}-5 \mathrm{SEQ}$ and $\mathrm{pB}-3 \mathrm{SEQ}$ for piggyBac $5^{\prime}$ and $3^{\prime}$ ends, respectively, and XP-5SEQ and XP-3SEQ for XP $5^{\prime}$ and $3^{\prime}$ ends, respectively. All primer sequences are available on request.

Association with genome and genes. The sequences recovered from transposon flanks were trimmed for quality and masked of any vector. We assembled $5^{\prime}$ and $3^{\prime}$ ends assembled and searched them against the D. melanogaster genomic sequence deposited in GenBank. Eighty-nine percent of all lines could be associated with a unique region of the genome. The remaining stocks either failed sequencing or could not be uniquely placed on the genome (sequence too short, repetitive, chimeric).

Availability of reagents and accessions. We made the fly strains described in this report available to public stock centers. The Bloomington Drosophila Stock Center selected $\sim 2,100$ stocks (Supplementary Table 2 online) to complement their existing collection. Harvard University (S. Artavanis-Tsakonas, Department of Cell Biology, Harvard Medical School, Charlestown, Massachusetts, USA) selected $\sim 16,500$ stocks (Supplementary Table 3 online), which were culled from the total to reduce redundant hits and provide a diversity of insert type across the genome. Stocks deposited at Harvard University may be requested by e-mail (drosophila@hms.harvard.edu). Resource constraints did not allow either repository to accept all the stocks reported here. We deposited genomic DNA sequence flanking insertion sites (Supplementary Tables $\mathbf{2}$ and $\mathbf{3}$ online) with the Berkeley Drosophila Genome Project and vector maps and plasmid DNA with the Drosophila Genomics Resource Center.

URLs. The method for obtaining flanking genomic sequence from $5^{\prime}$ and $3^{\prime}$ piggyBac ends by inverse PCR is available at http://www.fruitfly.org/about/ methods/inverse.pcr.html. The Bloomington Drosophila Stock Center is available at http://flystocks.bio.indiana.edu. The Drosophila Genomics Resource Center is available at http://dgrc.cgb.indiana.edu.

GenBank accession numbers. Vector sequences for PB, AY515146; RB, AY515147; WH, AY515148; and XP, AY515149.

Note: Supplementary information is available on the Nature Genetics website.

\section{ACKNOWLEDGMENTS}

We thank the many other people at Exelixis whose intellectual and technical contributions made this work possible, particularly our colleagues in the Genomics, Informatics and Genetics departments.

\section{COMPETING INTERESTS STATEMENT}

The authors declare competing financial interests (see the Nature Genetics website for details)

Received 2 October 2003; accepted 29 January 2004

Published online at http://www.nature.com/naturegenetics/

1. Adams, M.D. \& Sekelsky, J.J. From sequence to phenotype: reverse genetics in Drosophila melanogaster. Nat. Rev. Genet. 3, 189-198 (2002). 
2. Spradling, A.C. et al. The BDGP gene disruption project: single $P$ element insertions mutating 25\% of vital Drosophila genes. Proc. Natl. Acad. Sci. USA 153, 135-177 (1999).

3. Adams, M.D. et al. The Genome Sequence of Drosophila melanogaster. Science $\mathbf{2 8 7}$ 2185-2195 (2000).

4. Handler, A.M. \& Harrell, R.A.I. Germline transformation of Drosophila melanogaster with the piggyBac transposon vector. Insect Mol. Biol. 8, 449-457 (1999).

5. Handler, A.M. A current perspective on insect gene transformation. Insect Biochem. Mol. Biol. 31, 111-128 (2001).

6. Horn, C., Offen, N., Nystedt, S., Häcker, U. \& Wimmer, E.A. piggyBac-based insertional mutagenesis and enhancer detection as a tool for functional insect genomics. Genetics 163, 647-661 (2003).

7. Hacker, U., Nystedt, S., Barmchi, M.P., Horn, C. \& Wimmer, E.A. piggyBac-based insertional mutagenesis in the presence of stably integrated $P$ elements in Drosophila. Proc. Natl. Acad. Sci. USA 100, 7720-7725 (2003).

8. Zhang, P. \& Spradling, A.C. Efficient and dispersed local $P$ element transposition from Drosophila females. Genetics 133, 361-373 (1993).

9. Ashburner, M.A. Drosophila: a Laboratory Handbook (Cold Spring Harbor Laboratory Press, New York, 1989).

10. Cooley, L., Kelley, R. \& Spradling, A.C. Insertional mutagenesis of the Drosophila genome with single Pelements. Science 239, 1121-1128 (1988).

11. Rørth, P. A modular misexpression screen in Drosophila detecting tissue-specific phenotypes. Proc. Natl. Acad. Sci. USA 93, 12418-12422 (1996).

12. Spradling, A.C. et al. Gene disruptions using $P$ transposable elements: an integra component of the Drosophila genome project. Proc. Natl. Acad. Sci. USA 92, 10824-10830 (1995).

13. Horowitz, H. \& Berg, C.A. Aberrant splicing and transcriptional termination caused by $P$ element insertion into the intron of a Drosophila gene. Genetics 139, 327-335 (1995).

14. Goodwin, S.F. et al. Aberrant splicing and altered spatial expression patterns in fruit- less mutants of Drosophila melanogaster. Genetics 154, 725-745 (2000).

15. Cho, N. et al. Developmental control of blood cell migration by the Drosophila VEGF pathway. Cell 108, 865-876 (2002).

16. Gottar, M. et al. The Drosophila immune response against Gram-negative bacteria is mediated by a peptidoglycan recognition protein. Nature 416, 640-644 (2002).

17. Pirrotta, V. Vectors for $P$ mediated transformation in Drosophila. in Vectors: A Survey of Molecular Cloning Vectors and Their Uses (eds. R.L. Rodriquez \& D.T. Denhart) 437-456 (Butterworth, Boston, 1988).

18. Sadowski, P. The Flp recombinases of the 2-microns plasmid of Saccharomyces cerevisiae. Prog. Nucleic Acid Res. Mol. Biol. 51, 53-91 (1995).

19. Kim, Y.J., Zuo, P., Manley, J.L. \& Baker, B.S. The Drosophila RNA-binding protein RBP1 is localized to transcriptionally active sites of chromosomes and shows a functional similarity to human splicing factor ASF/SF2. Genes Dev. 6, 2569-2579 (1992).

20. Roseman, R.R. et al. A P element containing suppressor of Hairy-wing binding regions has novel properties for mutagenesis in Drosophila melanogaster. Genetics 141, 1061-1064 (1995).

21. Parks, A.L. et al. Systematic generation of high-resolution deletion coverage of the Drosophila melanogaster genome. Nat. Genet. advance online publication, 22 February 2004 (doi:10.1038/ng1312).

22. Golic, K.G. \& Lindquist, S. The FLP recombinase of yeast catalyzes site-specific recombination in the Drosophila genome. Cel/ 59, 499-509 (1989).

23. Theurkauf, W.E., Baum, H., Bo, J. \& Wensink, P.C. Tissue-specific and constitutive $\alpha$ tubulin genes of Drosophila melanogaster code for structurally distinct proteins. Proc. Natl. Acad. Sci. USA 83, 8477-8481 (1986).

24. Serano, T.L. \& Cohen, R.S. A small predicted stem-loop structure mediates oocyte localization of Drosophila K10 mRNA. Development 121, 3809-3818 (1995).

25. Rørth, P. Gal4 in the Drosophila female germline. Mech. Dev. 78, 113-118 (1998).

26. Robertson, H.M. et al. A stable genomic source of $P$ element transposase in Drosophila melanogaster. Genetics 118, 461-470 (1988). 